\title{
Gait analysis on the condition of arm swing in healthy young adults
}

\author{
Hyun-Min Koo, Su-Young Lee \\ Department of Physical Therapy, Division of Health Science, Baekseok University, Cheonan, Republic of Korea
}

Objective: The arm swing is associated with gait ability in healthy young adults. The purpose of this study was to examine the effects of arm swing during gait in healthy young adults.

Design: Cross-sectional study.

Methods: Forty-five subjects without any orthopedic or neurological injuries participated in this study. All subjects performed all three conditions according to the arm swing type as follows: first procedure (condition 1), walking as usual without arm swing constraint; second procedure (condition 2), constraint of dominant arm swing walking as usual; third procedure (condition 3), constraint of both arm swing walking as usual. Gait parameters such as gait velocity, stride length, cadence, step time, single limb support, and double limb support were measured in all arm swing conditions performed randomly, with the mean value obtained from three measurements. A rest period of 5 minutes was given to prevent repetition of each condition and learning effect. All data was analyzed using repeated measures ANOVA to notice the changes between arm swing conditions.

Results: Within walking conditions, significant difference of gait velocity, stride length, cadence, and double limb support was noticed $(p<0.05)$, except step time and single limb support. Gait velocity and stride length were significant reduced, and in cadence and double limb support were increased $(p<0.05)$. Condition 3 had the most significant decrease of gait ability compared with condition $1(p<0.05)$.

Conclusions: These finding suggested that constraint arm swing conditions reduced gait ability in healthy young adults. Also, these findings can be utilized as a reference to future studies that not only pelvic, knee and ankle, but also upper limb affect to gait ability.

Key Words: Arm, Gait, Young adult

\section{Introduction}

Gait is the most basic behavior of humans, and it is a form of motion in moving the body forward through repetitive exercise [1]. Also the nervous system, musculoskeletal system, and physiological support systems are all functionally integrated yet organically interdependent [2]. Normal gait is a complicated activity that requires interaction between more than two joints of the lower limb, body, the upper limb, and the lower limb in harmonious movement effectively moving the center of gravity of the body using minimum energy consumption and maintaining stability in standing po- sition $[3,4]$.

Gait analysis provides a scientific basis to understand the pathological walking mechanism and is used to determine accurately the systematic treatment and subsequent effect through objective and quantitative evaluation [5]. Also clinically, usability of gait analysis has gradually increased for assessment before treatment, surgery decision, and re-examination after surgery of adults and young children [6]. The natural movement of upper limb occurs during gait, then the arm swing and lower limb swing appear to move reciprocally [7]. These movements are not simply passive pendulum movements but have a feature that affects gait [8].

Received: 17 August, 2016 Revised: 6 September, 2016 Accepted: 6 September, 2016

Corresponding author: Su-Young Lee

Department of Physical Therapy, Division of Health Science, Baekseok University, 76 Munam-ro, Dongnam-gu, Cheonan 31065 , Republic of Korea Tel: 82-41-550-2546 Fax: 82-41-550-2829 E-mail: rosei118@bu.ac.kr

(c) This is an Open-Access article distributed under the terms of the Creative Commons Attribution Non-Commercial License (http://creativecommons.org/licens es/by-nc/4.0) which permits unrestricted non-commercial use, distribution, and reproduction in any medium, provided the original work is properly cited.

Copyright @ 2016 Korean Academy of Physical Therapy Rehabilitation Science 
Reciprocal arm swing is a typical feature of human walking [9]. Also some research give reason to why we swing arms during gait, which include minimizing energy consumption, optimizing stability, and optimizing neural performance [10].

Meyns et al. [10] suggested the reason why arm swing has direct affect to propulsion was unclear, due to human's bipedal walking. And as a result, most studies on gait tend to ignore arm swing altogether and suggested the arm swing was entirely passive, as a consequence of the movements of the thorax, gravity, and inertia [10]. But Elftman [8] concluded that since net moments in the shoulder joints occur, arm swing during gait is not passive, and driven by muscle activity.

Kim and Kwon [11] performed a gait analysis study that compared constraint arm swing condition and emphasis on arm swing condition for hemiplegic patients caused by stroke. Like this, most studies have been performed on patients. Among the studies for healthy adults, Ford et al. [12] studied the comparison of arm swing as usual and constraint arm swing about gait analysis for normal adults. Like this, the studies about arm swing have been progressed here and abroad, but they performed to examine the adaptive changes in coordination patterns. So this study examines the arm swing affecting gait ability when the arm swing was restricted. There has been a lack of evidence on the arm swing affecting gait ability for normal adults, because the studies about gait for normal adults were performed ignoring arm swing.

Thus, this study measures gait parameters according to constraint arm swing condition, walking as usual condition for normal adults and aims to know the change of gait ability using measured gait parameters. Through this study's outcome, this study will be utilized as a reference to future studies that not only pelvic, knee and ankle, but also upper limb affect to gait ability.

\section{Methods}

\section{Subjects}

The subjects of the study consisted of 45 healthy male and female adults at Baekseok University in Cheonan, South Korea. All subjects agreed to participate in the experiment after listening to the purpose and method and signed the consent. The subjects were selected according to the criteria as follows: those without orthopedic injuries to upper limb or lower limb for the past 6 months, those without neuro-
Table 1. General characteristics of the subjects $(\mathrm{N}=45)$

\begin{tabular}{lc}
\hline \multicolumn{1}{c}{ Category } & Mean (SD) \\
\hline No. of gender (male/female) & $15 / 30$ \\
Age $(\mathrm{y})$ & $20.8(1.6)$ \\
Height $(\mathrm{cm})$ & $165.5(8.2)$ \\
Weight $(\mathrm{kg})$ & $58.7(9.6)$ \\
Length of legs (right/left) & $85.1(5.3) / 85.2(5.3)$ \\
\hline
\end{tabular}

logical injuries affect to gait, and those who can understand and follow the instructions. The exclusion criteria included any vestibular system dysfunction and those who wished to give up during the experiment. The general characteristics of the research subjects are as Table 1.

\section{Experimental procedure}

All subjects of this study repeatedly performed in three conditions.

\section{Equipment}

\section{Gait analysis system}

To find out objective differences of gait in each condition, GAITRite system (CIR Systems Inc., Peekskill, NY, USA) was used as a tool for measuring spatiotemporal parameters. This device is a mat with an active sensor area of $3.7 \times 0.6 \mathrm{~m}$. When the subject walks along the mat, the metro computer senses the footprint and then gathers information about spatiotemporal parameters. The information about collected spatiotemporal parameters were processed using GAITRite GOLD, version 3.2b software (CIR Systems Inc.).

\section{Measurements}

\section{Arm swing condition during gait}

\section{Arm swing walking as usual (condition 1)}

Measured gait parameter with guiding the subjects verbally to walk as neutral as possible.

\section{Walking as usual with constraint dominant arm swing (condition 2)}

Measured gait parameter as the subjects had the dominant arm bent and crossed over the chest which was fixed by a strap then guided to walk swinging the other arm unfixed.

Walking as usual with constraint both arm swing (condition 3)

Measured gait parameter as the subjects had the both arms 
Table 2. Gait analysis on the condition of the arm swing

\begin{tabular}{lccccc}
\hline & Condition 1 & Condition 2 & Condition 3 & F & $p$-value \\
\hline Velocity (cm/s) & $136.85(15.9)$ & $129.19(14.90)^{\mathrm{a}}$ & $125.8(14.61)^{\mathrm{a}, \mathrm{b}}$ & 17.94 & $<0.01^{*}$ \\
Cadence (step/min) & $120.5(6.63)$ & $121.28(6.18)$ & $122.91(7.53)^{\mathrm{a}, \mathrm{b}}$ & 5.05 & $0.011^{*}$ \\
Stride length (cm) & $134.12(12.96)$ & $128.65(13.52)^{\mathrm{a}}$ & $124.86(12.47)^{\mathrm{a}, \mathrm{b}}$ & 34.37 & $<0.01^{*}$ \\
Step time (s) & $0.495(0.411)$ & $0.499(0.302)$ & $0.498(0.346)$ & 0.104 & 0.902 \\
Sing limb support (\%) & $39.36(4.15)$ & $38.60(4.11)$ & $38.39(3.02)$ & 2.12 & 0.13 \\
Double limb support (\%) & $20.95(4.47)$ & $22.83(5.67)^{\mathrm{a}}$ & $22.46(3.54)^{\mathrm{a}}$ & 4.02 & $0.02^{*}$ \\
\hline
\end{tabular}

Values are presented as mean (SD).

Condition 1, walking comfortable as usual; Condition 2, walking with constraint dominant arm swing; Condition 3, walking with constraint both arm swing.

${ }^{\mathrm{a}}$ Significantly different from Condition $1\left({ }^{*} p<0.05\right)$, ${ }^{\mathrm{b}}$ Significantly different from Condition $2\left({ }^{*} p<0.05\right)$.

bent and crossed over the chest which was fixed by a strap, then walked.

\section{Gait parameters}

Gait parameters that were used in this study were velocity, cadence, stride length, step time, single limb support, and double limb support. Velocity is dividing the distance traveled by ambulation time. It was expressed in centimeters per second $(\mathrm{cm} / \mathrm{s})$. Cadence is dividing the distance traveled by the step count. It was expressed in step per minute (step/min). Stride length is measured on the line of progression between the heel points of two consecutive footprints of the same foot. The unit of measure is centimeters. Step time is the time elapsed from first contact of one foot to first contact of the opposite foot. It is measured in seconds. Single limb support is the time elapsed between the last contact of the current footfall to the first contact of the next footfall of the same foot. Double limb support occurs from heel contact of one footfall to toe-off of the opposite footfall.

Prior to the experiment, thorough explanation and demonstration of the methods was given. And a guided verbal cue of "walking comfortable as usual" to the subjects in was given for each condition. When fixing the subject's arm, a measure of gait parameters was taken with the upper and lower part of the upper extremity including the elbow joint using a strap [11]. For collecting information, all conditions were repeatedly measured three times an average was used as a result value. A rest period of 5 minutes was given to prevent repetition of each condition and learning effect.

\section{Data analysis}

Collected data was analyzed by using PASW Statistics ver. 18.0 for Windows (IBM Co., Armonk, NY, USA). It was used for descriptive statistic for the general characteristics

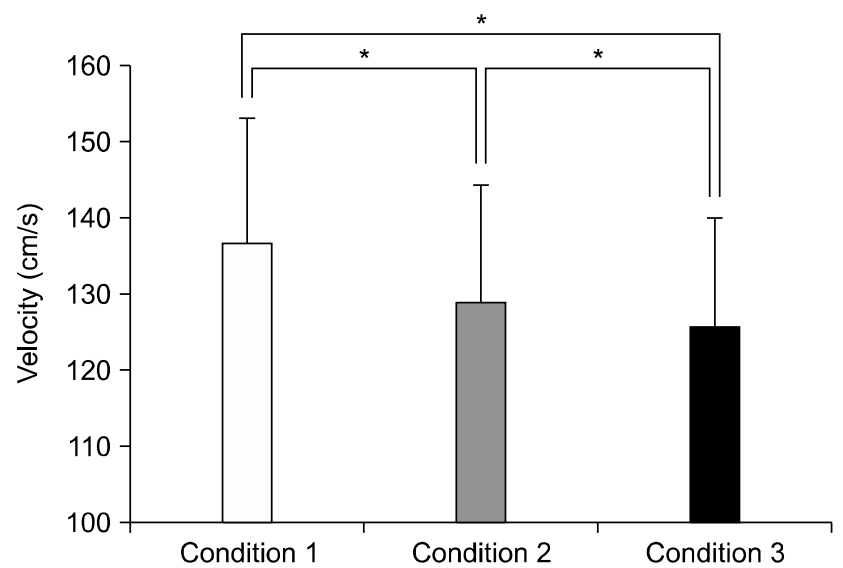

Figure 1. Gait velocity on the condition of the arm swing. Condition 1, walking comfortable as usual; Condition 2, walking with constraint dominant arm swing; Condition 3, walking with constraint both arm swing. ${ }^{*} p<0.05$.

(age, height, weight, and length of legs). In order to compare mean differences of the three conditions, repeated measures ANOVA was used. The statistical significant level $(\alpha)$ for data treatment was set at 0.05 .

\section{Results}

\section{Gait analysis on the conditions of arm swing}

Results showing differences of spatiotemporal parameters like velocity, cadence, stride length, step time, single limb support, and double limb support in the three conditions (walking comfortable as usual, walking with constraint dominant arm swing, walking with constraint both arm swing) is shown in Table 2. The mean difference of condition 1 and 2 was $7.6 \mathrm{~cm} / \mathrm{s}$, also condition 1 and 3 was 11.05 $\mathrm{cm} / \mathrm{s}$ in velocity. Thus showing significant decrease in all constraint arm swing conditions compared with walking as 


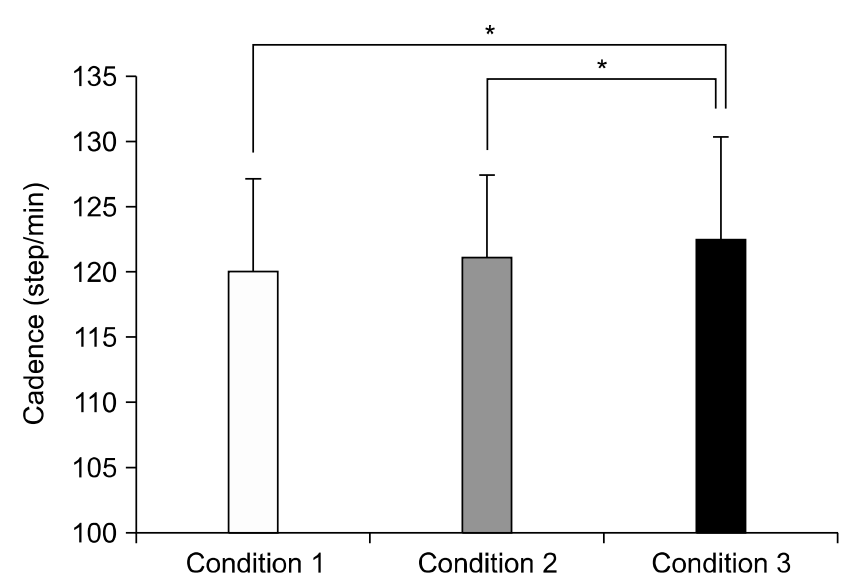

Figure 2. Cadence on the condition of the arm swing. Condition 1, walking comfortable as usual; Condition 2, walking with constraint dominant arm swing; Condition 3, walking with constraint both arm swing. ${ }^{*} p<0.05$.

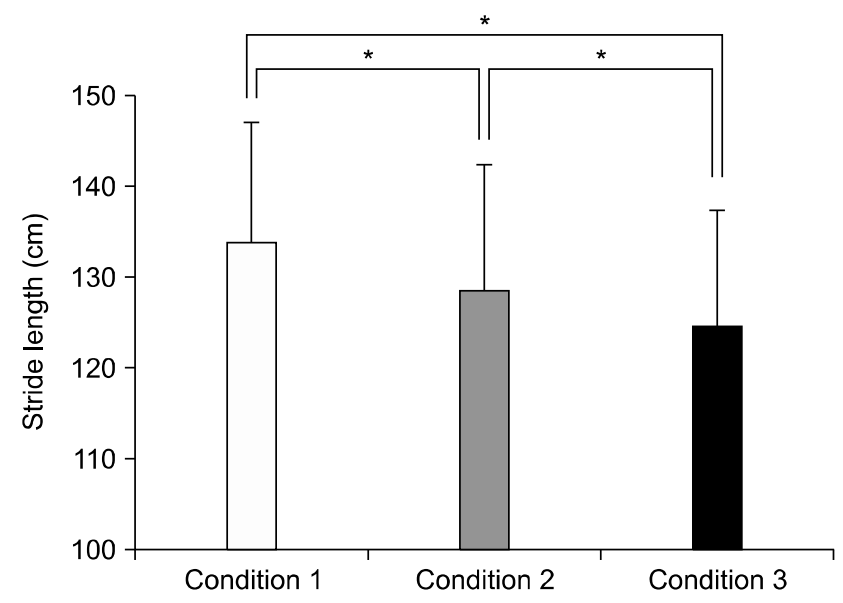

Figure 3. Stride length on the condition of the arm swing. Condition 1, walking comfortable as usual; Condition 2, walking with constraint dominant arm swing; Condition 3, walking with constraint both arm swing. ${ }^{*} p<0.05$.

usual condition ( $p<0.05$; Figure 1). In cadence, the mean difference of condition 1 and 3 were $-2.41 \mathrm{step} / \mathrm{min}$, also condition 2 and 3 was $-1.63 \mathrm{step} / \mathrm{min}$. Thus there was a significant increase in condition 3 compared with condition 2 and $3(p<0.01$; Figure 2$)$. But there was not a significant difference between condition 1 and $2(p>0.05)$, and in stride length, the mean difference of condition 1 and 2 was 5.46 $\mathrm{cm}$, also condition 1 and 3 was $9.25 \mathrm{~cm}$. Thus there was a significant decrease in all constraint arm swing conditions compared with walking as usual condition $(p<0.05$; Figure 3 ). In double limb support, the mean difference of condition 1 and 2 were $-1.88 \%$, also condition 1 and 3 were $-1.50 \%$. Thus there was a significant increase in condition 2 and 3

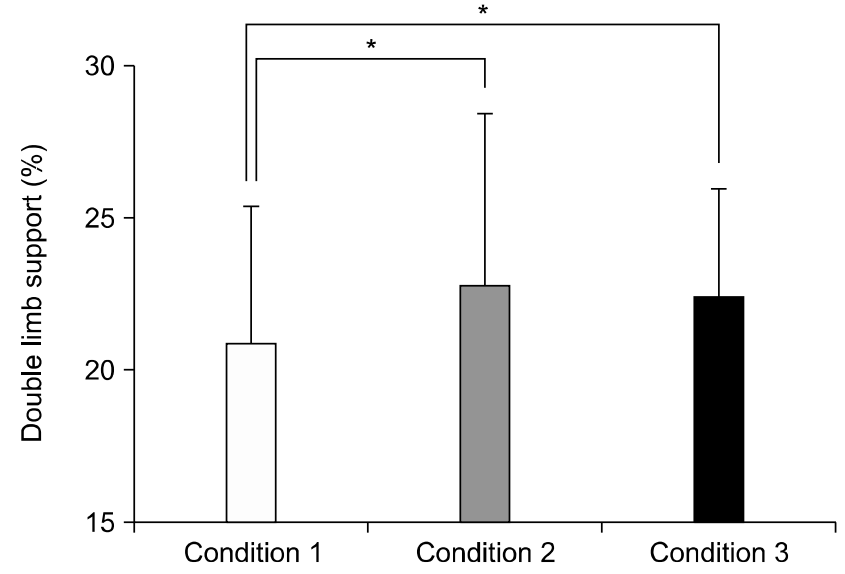

Figure 4. Double limb support on the condition of the arm swing. Condition 1, walking comfortable as usual; Condition 2, walking with constraint dominant arm swing; Condition 3, walking with constraint both arm swing. ${ }^{*} p<0.05$.

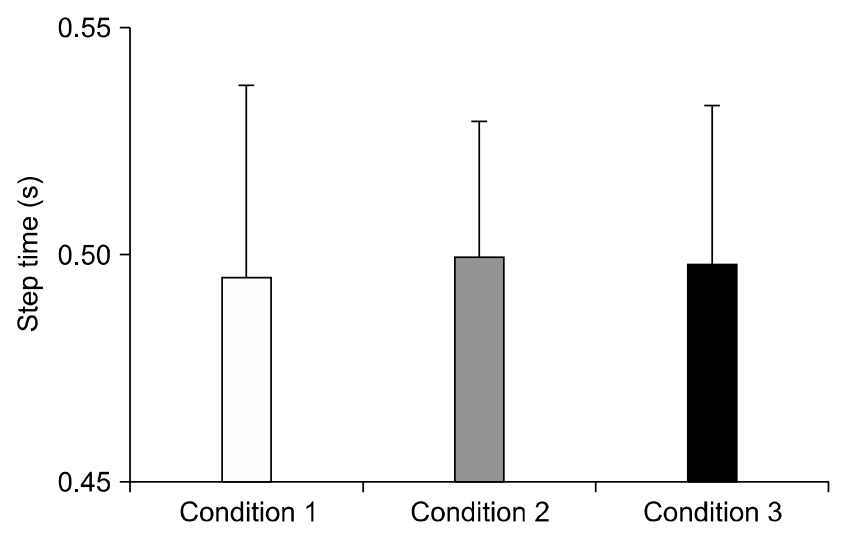

Figure 5. Step time on the condition of the arm swing. Condition 1 , walking comfortable as usual; Condition 2, walking with constraint dominant arm swing; Condition 3, walking with constraint both arm swing.

compared with condition 1 (Figure 4). Other gait parameters such as step time and single limb support did not show significant differences ( $p>0.05$; Figures 5, 6).

\section{Discussion}

The aim of the present study was to investigate effect of arm swing to gait ability to healthy young adults in their twenties. The results showed that gait ability with constraint arm swing conditions was decreased versus walking as usual condition.

Kubo et al. [13] reported that velocity increased because of increasing rotation between the thorax and pelvic through 


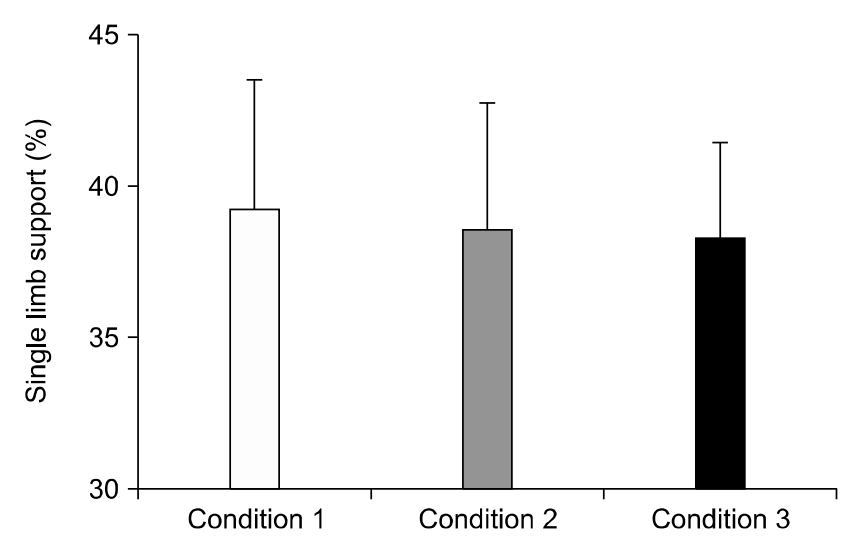

Figure 6. Single limb support on the condition of the arm swing. Condition 1, walking comfortable as usual; Condition 2, walking with constraint dominant arm swing; Condition 3, walking with constraint both arm swing.

the arm swing. And Ford et al. [12] reported that velocity was decreased because of decreasing coordination of movements between upper limb and lower limb when restricting the upper limbs movement during gait. Similar research reported differences in value of walking speed, power value of joint, and ground reaction force when performing the experiment in normal arm swing condition, restricted arm swing condition, and fitness arm swing condition [14]. In this study, velocity and stride length decreased when the dominant arm was fixed, and both arms, compared to an unfixed arm condition. It was considered that the reason why velocity was reduced is that decrease of propulsion was due to restriction of arm swing during gait.

Referring to studies involved in the arm swing, Umberger [15] reported that the role of upper limbs during gait maintained balance of the body when the rotation of the pelvis in transverse plane has been transmitted to the upper body because of compensation due to rotation in opposite arms. Therefore, stroke patients have asymmetric posture and balance disorder due to hemiplegia [16]. Also restricting upper limb's movement during gait causes changes in walking pattern due to compensation occurring in various forms [12]. In this study, the subjects were focused not on losing their balance in increased movement of the pelvis and trunk instead of arm swing to overcome the difficulty of walking due to the restriction of arm swing. Because of this, it was considered that velocity and stride length decreased in constraint arm swing conditions compared with walking as usual condition.

Eke-Okoro et al. [17] reported that when constraint arm movement was compared to no arm constraint in normal subjects, stride length, stride frequency, and walking velocity decreased. In this study, cadence was increased on arm constraint condition, while stride and walking velocity decreased on arm constraint condition compared to no arm constraint. This result means that cadence compensates for their shorter stride length. Ford et al. [12] described that when carrying a load with the hands, in healthy adults cadence increase can overcome decreased transverse rotation of trunk and pelvis, and stride length.

But this study has several limitations. Firstly, the selection criteria of the subjects were young adults, so only young adults in their twenties were selected. Therefore, it is difficult to generalize research results to various age groups. Secondly, this study was performed in a single condition without variety of walking conditions, which resulted in common outcomes. Lastly, it is difficult to give the affect to overall gait ability because the number of gait parameters used in gait analysis was minimal. Thus studies selecting various age groups for the subjects using more gait parameters and various walking conditions will be required to increase reliability about gait analysis in the future.

This study attempted to examine the effect of arm swing to gait ability to healthy young adults in their twenties. According to constraint condition of arm swing through gait analysis, gait ability happened to decrease when the subjects had walked with constraint arm swing. It will be utilized as a reference to future studies that not only the pelvis, knee and ankle, but also upper limb affects gait ability.

\section{Conflict of Interest}

The authors declared no potential conflicts of interest with respect to the authorship and/or publication of this article.

\section{References}

1. Park JY, Jin YW. The analysis of gait pattern about 20 ' adult male and female. Eronon Soc Korea 2009;11:466-9.

2. Kim G, Yoon NM. A study on kinetic gait analysis of the normal adult. J Korean Soc Phys Ther 2009;21:87-95.

3. Lee HK, Lee HH, Park YM, Lee JH, Ha TY. Regulation of human B cell proliferation and differentiation by seminal plasma. Clin Exp Immunol 1991;85:174-9.

4. Jung HS. Analysis of kinematic parameters of gait in normal subject. J Korea Acad-Ind Cooper Soc 2014;15:2989-95.

5. Schutte LM, Narayanan U, Stout JL, Selber P, Gage JR, Schwartz $\mathrm{MH}$. An index for quantifying deviations from normal gait. Gait Posture 2000;11:25-31. 
6. Yoon NM, Yoon HJ, Park JS, Jeong HS, Kim G. The comparative study on age-associated gait analysis in normal Korean. J Korean Soc Phys Ther 2010;22:15-24.

7. Wagenaar RC, van Emmerik RE. Resonant frequencies of arms and legs identify different walking patterns. J Biomech 2000;33: 853-61.

8. Elftman H. The function of the arms in walking. Human Biol 1939;11:529-535.

9. Kuhtz-Buschbeck JP, Jing B. Activity of upper limb muscles during human walking. J Electromyogr Kinesiol 2012;22:199-206.

10. Meyns P, Bruijn SM, Duysens J. The how and why of arm swing during human walking. Gait Posture 2013;38:555-62.

11. Kim JS, Kwon OH. The effect of arm swing on gait in post-stroke hemiparesis. J Korean Soc Phys Med 2012;7:95-101.

12. Ford MP, Wagenaar RC, Newell KM. Arm constraint and walk- ing in healthy adults. Gait Posture 2007;26:135-41.

13. Kubo M, Holt KG, Saltzman E, Wagenaar RC. Changes in axial stiffness of the trunk as a function of walking speed. J Biomech 2006;39:750-7.

14. Jung HS, Choi SH, Park SJ, Oh HJ, Cho HY. The effect of arm swing on gait in healthy adults. J Dig Converg 2014;12:451-9.

15. Umberger BR. Effects of suppressing arm swing on kinematics, kinetics, and energetics of human walking. J Biomech 2008;41: 2575-80.

16. Kim YC, Jang SJ, Park MY, Park SW. Prognostic factors of ambulation in stroke patients. J Korean Acad Rehab Med 1992;16: 443-51.

17. Eke-Okoro ST, Gregoric M, Larsson LE. Alterations in gait resulting from deliberate changes of arm-swing amplitude and phase. Clin Biomech (Bristol, Avon) 1997;12:516-21. 